\title{
Effect of long-term underfeeding and subsequent refeeding on hay digestibility in sheep
}

\author{
R Perrier, M Doreau
}

\author{
INRA, Laboratoire Sous-Nutrition des Ruminants, Theix, 63122 St-Genès-Champanelle, France
}

Decreasing the level of intake generally increases diet digestibility, mainly because of an increase in particle retention time in the rumen (Galyean and Owens, 1991, in Physiological Aspects of Digestion and Metabolism in Ruminants, Acad Press, San Diego, USA). In most experiments, the effect of intake on digestibility is measured in short-term trials, after an adaptation period of 3 or 4 weeks. A long-term trial has been carried out by Grimaud and Doreau (1995, J Anim Sci, 73, 211-219), but in this experiment decrease in intake did not result in variations of digestibility. The aim of the present trial was thus to examine the consequences of a long-term underfeeding followed by a realimentation on diet digestibility.

Six adult Lacaune ewes, weighing on average $60 \mathrm{~kg}$ at the beginning of the experiment, received a diet exclusively made of a regrowth of natural grassland hay, containing $90.8 \%$ organic matter, $62.9 \%$ NDF, $35.1 \%$ ADF and $14.4 \%$ crude protein. Animals were first fed at the level of maintenance requirement for 4 weeks, then at $0.5 \times$ requirement level for 10 weeks, thereafter returned to the initial feeding level. Digestibility was measured by total collection for 5 days at the end of the first phase (P1), after 3 (P2) and 9 (P3) weeks of underfeeding and during the third week after realimentation $(\mathrm{P} 4)$.

Decreasing intake resulted in an increase in digestibility of hay : total fibre digestibility increased more than organic matter digestibility (12.5 vs 4.1 percentage units) and more than generally observed with all-hay diets (Kabré et al, 1995, J Agric Sci, 124, 119-127). No significant difference occurred between P2 and P3, suggesting that the adaptation to a low feed intake was rapidly achieved, perhaps because of the rapid adaptation of digestive organs to variations of food intake (review by Ortigues and Doreau, 1995, Ann Zootech, 44, in press). After realimentation, digestibility significantly increased, more for total fibre than for organic matter ( 6.9 vs 4.9 percentage units). This result is very surprising because very few literature data showed an increase in digestibility when intake increases.

However, one of these experiments (Gingins et al, 1980, Livest Prod Sci, 7, 465-471) exhibited such a variation when sheep were realimented after an underfeeding period of 15 weeks. In the present experiment a specific effect of the quality of hay on digestibility cannot be excluded; however it is not likely: the apparent quality of hay, estimated from NDF and crude protein contents, was not improved in $\mathrm{P} 4$.

Further research is needed about the effect of sequential effects of underfeeding and realimentation, accompanied by measurements of retention time and microbial activity in the rumen, in order to explain the origin of variations of digestibility.

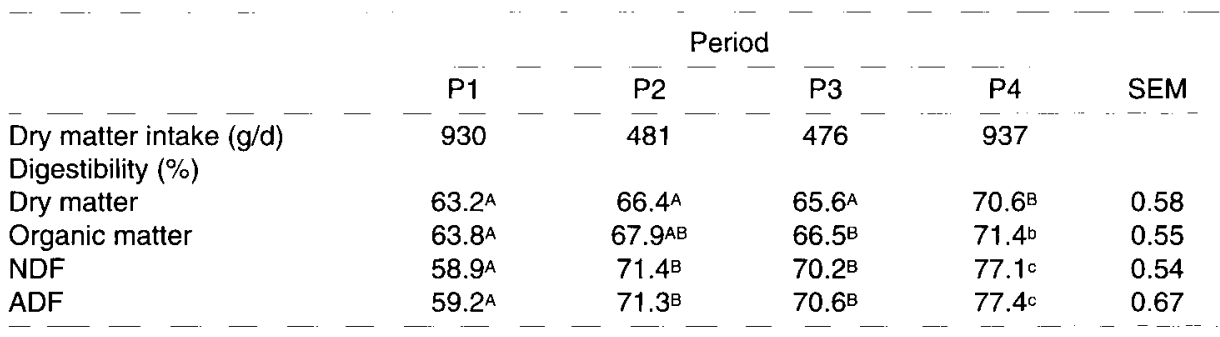

$\bar{M}$ eans on the same row with different superscripts significantly differ $\overline{(P}<0 . \overline{05})$ 